\title{
New Associative Memories to Recall Real-Valued Patterns
}

\author{
Humberto Sossa, Ricardo Barrón, and Roberto A. Vázquez \\ Centro de Investigación en Computación-IPN \\ Av. Juan de Dios Bátiz, esquina con Miguel Othón de Mendizábal \\ Mexico City, 07738, Mexico \\ hsossa@cic.ipn.mx
}

\begin{abstract}
In this note we describe a new set of associative memories able to recall patterns in the presence of mixed noise. Conditions are given under which the proposed memories are able to recall patterns either from the fundamental set of patterns and from distorted versions of them. Numerical and real examples are also provided to show the efficiency of the proposal.
\end{abstract}

\section{Introduction}

An associative memory is a device designed to recall patterns. These patterns might appear altered by noise. An associative memory $\mathbf{M}$ can be viewed as an input-output system as follows: $\mathbf{x} \rightarrow \mathbf{M} \rightarrow \mathbf{y}$, with $\mathbf{x}$ and $\mathbf{y}$, respectively the input and output patterns vectors. Each input vector forms an association with a corresponding output vector. The associative memory $\mathbf{M}$ is represented by a matrix whose $i j$-th component is $m_{i j} . \mathbf{M}$ is generated from a finite a priori set of known associations, known as the fundamental set of associations, or simply the fundamental set (FS). If $\xi$ is an index, the fundamental set is represented as: $\left\{\left(\mathbf{x}^{\xi}, \mathbf{y}^{\xi}\right) \mid \xi=1,2, \ldots, p\right\}$ with $p$ the cardinality of the set. The patterns that form the fundamental set are called fundamental patterns. If it holds that $\mathbf{x}^{\xi}=\mathbf{y}^{\xi} \forall \xi \in\{1,2, \ldots p\}$, then $\mathbf{M}$ is auto-associative, otherwise it is hetero-associative. A distorted version of a pattern $\mathbf{x}$ to be recuperated will be denoted as $\widetilde{\mathbf{x}}$. If when feeding a distorted version of $\mathbf{x}^{w}$ with $w \in\{1,2, \ldots, p\}$ to an associative memory $\mathbf{M}$, then it happens that the output corresponds exactly to the associated pattern $\mathbf{y}^{w}$, we say that recalling is perfect. Several models for associative memories have emerged in the last 40 years. Refer for example to [1-4].

\section{Foundations of the Proposed Memories}

Let $P=\left\lfloor p_{i j}\right\rfloor_{m \times r}$ and $Q=\left\lfloor q_{i j}\right\rfloor_{r \times n}$ two matrices.

Definition 1. The following two matrix operations are defined to recall integervalued patterns:

1. Operation $\diamond_{\mathrm{A}}: P_{m \times r} \diamond_{\mathrm{A}} Q_{r \times n}=\left\lfloor f_{i j}^{\mathrm{A}}\right\rfloor_{m \times n}$ where $f_{i j}^{\mathrm{A}}=\bigotimes_{k=1}^{r} \mathrm{~A}\left(p_{i k}, q_{k j}\right)$. 
2. Operation $\diamond_{\mathrm{B}}: P_{m \times r} \diamond_{\mathrm{B}} Q_{r \times n}=\left[f_{i j}^{\mathrm{B}}\right]_{m \times n}$ where $f_{i j}^{\mathrm{B}}=\bigotimes_{k=1}^{r} \mathrm{~B}\left(p_{i k}, q_{k j}\right)$.

According to the operators $\otimes, \mathrm{A}$ and $\mathrm{B}$ used different results can be obtained. If we want, for example, to compensate for additive or subtractive noise, operator $\otimes$ should be replaced either by $\max (\vee)$ or $\min (\wedge)$ as in [4]. Median operator (med), should be adopted in the case of mixed noise. In this paper we use med operator because as we will show, it provides excellent results in the presence of mixed noise. It can be easily shown that if $\mathbf{x} \in \mathbf{Z}^{n}$ and $\mathbf{y} \in \mathbf{Z}^{m}$, then $\mathbf{y} \diamond_{\mathrm{A}} \mathbf{x}^{t}$ is a matrix of dimensions $m \times n$.

Relevant simplifications are obtained when operations $\diamond_{\mathrm{A}}$ and $\diamond_{\mathrm{B}}$ are applied between vectors:

1. If $\mathbf{x} \in \mathbf{Z}^{n}$ and $\mathbf{y} \in \mathbf{Z}^{m}$, then $\mathbf{y} \diamond_{\mathrm{A}} \mathbf{x}^{t}$ is a matrix of dimensions $m \times n$, and also it holds that

$$
\mathbf{y} \diamond_{\mathrm{A}} \mathbf{x}^{t}=\left(\begin{array}{cccc}
\mathrm{A}\left(y_{1}, x_{1}\right) & \mathrm{A}\left(y_{1}, x_{2}\right) & \cdots & \mathrm{A}\left(y_{1}, x_{n}\right) \\
\mathrm{A}\left(y_{2}, x_{1}\right) & \mathrm{A}\left(y_{2}, x_{2}\right) & \cdots & \mathrm{A}\left(y_{2}, x_{n}\right) \\
\vdots & \vdots & \ddots & \vdots \\
\mathrm{A}\left(y_{m}, x_{1}\right) & \mathrm{A}\left(y_{m}, x_{2}\right) & \cdots & \mathrm{A}\left(y_{m}, x_{n}\right)
\end{array}\right)_{m \times n} .
$$

2. If $\mathbf{x} \in \mathbf{Z}^{n}$ and $P$ a matrix of dimensions $m \times n$, operations $P_{m \times n} \diamond_{\mathrm{B}} \mathbf{x}$ gives as a result one vector with dimension $m$, with $i$-th component given as $\left(P_{m \times r} \diamond_{\mathrm{B}} \mathbf{x}\right)_{i}=\underset{j=1}{n} \underset{\operatorname{med}}{ } \mathrm{B}\left(p_{i j}, x_{j}\right)$.

If $\mathbf{x} \in \mathbf{Z}^{n}$ and $P$ a matrix of dimensions $m \times n$ then operation $\mathbf{M}_{m \times n} \diamond_{\mathrm{B}} \mathbf{x}$ outputs an $m$-dimensional column vector, with $i$-th component given as: $\left(\mathbf{M}_{m \times n} \diamond_{\mathrm{B}} \mathbf{x}\right)_{i}=\underset{j=1}{n} \mathbf{m} \mathrm{B}\left(m_{i j}, x_{j}\right)$.

Operators A and B might be chosen among those already proposed in the literature. In this paper we use operators A and B proposed in [4]. Operators A and B are defined as follows:

$$
\begin{aligned}
& \mathrm{A}(x, y)=x-y \\
& \mathrm{~B}(x, y)=x+y
\end{aligned}
$$

\section{Kinds of Noises}

The proposed memories can cope with several kinds of noises. Among them: additive, subtractive and mixed. In this paper, we are interested in leading with mixed noise. Let $\mathbf{x} \in \mathbf{R}^{n}$ be an input fundamental pattern to an associative memory. Pattern $\mathbf{x}$ can be altered or corrupted by mixed noise to produce a vector $\widetilde{\mathbf{x}}$ by adding or subtracting at random to each component of $\mathbf{x}, x_{i}$ a real $c, \widetilde{x}_{i}=x_{i}+c$ (additive noise), and $\widetilde{x}_{i}=x_{i}-c$ (subtractive noise). 


\section{The New Memories}

Two kind of associative memories are proposed, hetero-associative and autoassociative. Due to space, in this paper only hetero-associative memories are described. One hetero-associative memory is described: HS-memory of type $\mathbf{M}$.

\section{TRAINING PHASE:}

Step 1: For each $\xi=1,2, \cdots, p$, from each couple $\left(\mathbf{x}^{\xi}, \mathbf{y}^{\xi}\right)$ build matrix: $\left|\mathbf{y}^{\xi} \diamond_{\mathrm{A}}\left(\mathbf{x}^{\xi}\right)^{t}\right|_{m \times n}$.

Step 2: Apply the median operator to the matrices obtained in Step 1 to get matrix M as follows:

$$
\mathbf{M}=\underset{\xi=1}{p} \mathbf{m e d}^{\xi}\left[\mathbf{y}^{\xi} \diamond_{\mathrm{A}}\left(\mathbf{x}^{\xi}\right)^{t}\right] .
$$

The $i j$-th component $\mathbf{M}$ is given as follows:

$$
m_{i j}=\operatorname{med}_{\xi=1}^{p} \mathrm{~A}\left(y_{i}^{\xi}, x_{j}^{\xi}\right)
$$

\section{RECALLING PHASE:}

We have two cases, i.e.:

Case 1: Recall of a fundamental pattern. A pattern $\mathbf{x}^{w}$, with $w \in\{1,2, \cdots, p\}$ is presented to the memory $\mathbf{M}$ and the following operation is done:

$$
\mathbf{M} \diamond_{\mathrm{B}} \mathbf{x}^{w}
$$

The result is a column vector of dimension $n$, with $i$-th component given as:

$$
\left(\mathbf{M} \diamond_{\mathrm{B}} x^{w}\right)_{i}=\operatorname{med}_{j=1}^{n} \mathrm{~B}\left(m_{i j}, x_{j}^{w}\right) .
$$

Case 2: Recalling of a pattern from an altered version of it. A pattern $\widetilde{\mathbf{x}}$ (altered version of a pattern $\mathbf{x}^{w}$ is presented to the hetero-associative memory $\mathbf{M}$ and the following operation is done:

$$
\mathbf{M} \diamond_{\mathrm{B}} \widetilde{\mathbf{x}}
$$

Again, the result is a column vector of dimension $n$, with $i$-th component given as:

$$
\left(\mathbf{M} \diamond_{\mathrm{B}} \widetilde{\mathbf{x}}\right)_{i}=\operatorname{med}_{j=1}^{n} \mathrm{~B}\left(m_{i j}, \widetilde{x}_{j}\right) .
$$

Conditions, not proved here due to space, for perfect recall of a pattern of the FS or from altered version of them follow:

Theorem 1. Let $\left\{\left(\mathbf{x}^{\alpha}, \mathbf{y}^{\alpha}\right) \mid \alpha=1,2, \ldots, p\right\}$ with $\mathbf{x}^{\alpha} \in \mathbf{R}^{n}, \mathbf{y}^{\alpha} \in \mathbf{R}^{m}$ the fundamental set of an HS-memory $\mathbf{M}$ and let $\left(\mathbf{x}^{\gamma}, \mathbf{y}^{\gamma}\right)$ an arbitrary fundamental couple 
with $\gamma \in\{1, \cdots, p\}$. If $\operatorname{med}_{j=1}^{n} \varepsilon_{i j}=0, \quad i=1, \cdots, m, \quad \varepsilon_{i j}=m_{i j}-\mathrm{A}\left(y_{i}^{\gamma}, x_{j}^{\gamma}\right)$ then $\left(\mathbf{M} \diamond_{\mathrm{B}} \mathbf{x}^{\gamma}\right)_{i}=\mathbf{y}_{i}^{\gamma}, i=1 \ldots m$.

More restricted conditions are given by the following:

Corollary 1. Let $\left\{\left(\mathbf{x}^{\alpha}, \mathbf{y}^{\alpha}\right) \mid \alpha=1,2, \ldots, p\right\}, \mathbf{x}^{\alpha} \in \mathbf{R}^{n}, \mathbf{y}^{\alpha} \in \mathbf{R}^{m}$. A HA-median memory $\mathbf{M}$ has perfect recall if for all $\alpha=1, \cdots, p, \quad \mathbf{M}^{\alpha}=\mathbf{M}$ where $\mathbf{M}=\mathbf{y}^{\xi} \diamond_{\mathrm{A}}\left(\mathbf{x}^{\xi}\right)^{t}$ is the associated partial matrix to the fundamental couple $\left(\mathbf{x}^{\alpha}, \mathbf{y}^{\alpha}\right)$ and $p$ is the number of couples.

Theorem 2. Let $\left\{\left(\mathbf{x}^{\alpha}, \mathbf{y}^{\alpha}\right) \mid \alpha=1,2, \ldots, p\right\}, \mathbf{x}^{\alpha} \in \mathbf{R}^{n}, \mathbf{y}^{\alpha} \in \mathbf{R}^{m}$ a FS with perfect recall. Let $\eta^{\alpha} \in \mathbf{R}^{n}$ a pattern of mixed noise. A HA-median memory $\mathbf{M}$ has perfect recall in the presence of mixed noise if this noise is of median zero, this is if $\underset{j=1}{\operatorname{med}} \eta_{j}^{\alpha}=0, \forall \alpha$.

Example 1. The reader can easily verify that for the following set of patterns satisfies the conditions given by Theorem 1 and Corollary 1:

$$
\mathbf{x}^{1}=\left(\begin{array}{l}
0.1 \\
0.0 \\
0.2
\end{array}\right), \mathbf{y}^{1}=\left(\begin{array}{l}
0.2 \\
0.3 \\
0.3 \\
0.4
\end{array}\right) ; \mathbf{x}^{2}=\left(\begin{array}{l}
0.4 \\
0.3 \\
0.5
\end{array}\right), \mathbf{y}^{2}=\left(\begin{array}{l}
0.5 \\
0.6 \\
0.6 \\
0.7
\end{array}\right) \text { and } \mathbf{x}^{3}=\left(\begin{array}{l}
0.7 \\
0.6 \\
0.8
\end{array}\right), \mathbf{y}^{3}=\left(\begin{array}{l}
0.8 \\
0.9 \\
0.9 \\
1.0
\end{array}\right) .
$$

If a FS satisfies the conditions imposed by Theorem 1, and the noise added to a pattern $\mathbf{x}^{\alpha}$ of this FS satisfies Theorem 2, then no matter the level of noise added, the pattern is perfectly recalled. If this is the case you can easily prove that a given pattern $\mathbf{x}^{\alpha}$ is recalled through the information of the associated column of matrix $\mathbf{M} \diamond_{\mathrm{B}} \widetilde{\mathbf{x}}^{\alpha}$ of an element $x_{i}^{\alpha}$ of $\mathbf{x}^{\alpha}$ not affected by noise. Let us verify this with an example.

Example 2. Suppose we want to recall the first fundamental pattern from Example 1 given the following distorted version of its key:

$$
\widetilde{\mathbf{x}}^{1}=\left(\begin{array}{c}
0.3 \\
0.0 \\
-0.2
\end{array}\right) \text {. }
$$

As you can appreciate for the distorted pattern, the median of the noise added to $\mathbf{x}$ equals 0: $\operatorname{med}(0.2,0.0,-0.4)=0.0$. Also element number two of $\mathbf{x}^{1}$ is not affected by the added noise. Pattern $\mathbf{x}^{1}$ is thus recalled through the information of column two (underlined) associated to $x_{2}^{1}$ of $\mathbf{M} \diamond_{\mathrm{B}} \widetilde{\mathbf{x}}^{1}$. Let us verify this. 


\section{RECALLING PHASE:}

$\mathbf{M} \diamond_{\mathrm{B}} \mathbf{x}^{1}=\left(\begin{array}{ccc}0.1 & 0.2 & 0.0 \\ 0.2 & 0.3 & 0.1 \\ 0.2 & 0.3 & 0.1 \\ 0.3 & 0.4 & 0.2\end{array}\right) \diamond_{\mathrm{B}}\left(\begin{array}{c}0.3 \\ 0.0 \\ -0.2\end{array}\right)=\left(\begin{array}{c}\operatorname{med}[\mathrm{B}(0.1,0.3), \mathrm{B}(0.2,0.0), \mathrm{B}(0.0,-0.2)] \\ \operatorname{med}[\mathrm{B}(0.2,0.3), \mathrm{B}(0.3,0.0), \mathrm{B}(0.1,-0.2)] \\ \operatorname{med}[\mathrm{B}(0.2,0.3), \mathrm{B}(0.3,0.0), \mathrm{B}(0.1,-0.2)] \\ \operatorname{med}[\mathrm{B}(0.3,0.3), \mathrm{B}(0.4,0.0), \mathrm{B}(0.2,-0.2)]\end{array}\right)=\left(\begin{array}{c}\operatorname{med}(0.4, \underline{0.2},-0.2) \\ \operatorname{med}(0.5, \underline{0.3},-0.1) \\ \operatorname{med}(0.5, \underline{0.3},-0.1) \\ \operatorname{med}(0.6, \underline{0.4}, 0.0)\end{array}\right)=\left(\begin{array}{c}0.2 \\ 0.3 \\ 0.3 \\ 0.4\end{array}\right)$

\section{Case of a General Fundamental Set}

In practice most of the fundamental sets of patterns do not satisfy the restricted conditions imposed by Theorem 1 and its Corollary. If this not the case, we propose the following procedure to perfectly recall a general FS. Given a FS not satisfying Theorem 1:

\section{TRAINING PHASE:}

Step 1. Transform the FS into an auxiliary fundamental set (FS') satisfying Theorem 1:

1) Make $d=$ cont .

2) Make $\left(\overline{\mathbf{x}}^{1}, \overline{\mathbf{y}}^{1}\right)=\left(\mathbf{x}^{1}, \mathbf{y}^{1}\right)$.

3) For the remaining couples do \{

For $\xi=2$ to $p\{$

For $i=1$ to $n\{$

$$
\begin{aligned}
& \bar{x}_{i}^{\xi}=\bar{x}_{i}^{\xi-1}+d ; \hat{x}_{i}^{\xi}=\bar{x}_{i}^{\xi}-x_{i}^{\xi} ; \\
& \overline{\mathbf{y}}_{i}^{\xi}=\overline{\mathbf{y}}_{i}^{\xi-1}+d ; \hat{\mathbf{y}}_{i}^{\xi}=\mathbf{x}_{i}^{\xi}-\mathbf{x}_{i}^{\xi} .
\end{aligned}
$$$$
\}
$$

Step 2. Build matrix M in terms of set FS': Apply to FS' steps 1 and 2 of the training procedure described at the beginning of Section 2.3.

Remark 1. We can use any $d$. In this work we decided to use however the difference between the first components.

\section{RECALLING PHASE:}

We have also two cases, i.e.:

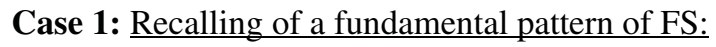

1) Transform $\mathbf{x}^{\xi}$ to $\overline{\mathbf{x}}^{\xi}$ by applying the following transformation: $\overline{\mathbf{x}}^{\xi}=\mathbf{x}^{\xi}+\hat{\mathbf{x}}^{\xi}$.

2) Apply equations (4) and (5) to each $\overline{\mathbf{x}}^{\xi}$ of FS' to recall $\overline{\mathbf{y}}^{\xi}$.

3) Recall each $\mathbf{y}^{\xi}$ by applying the following inverse transformation:

$$
\mathbf{y}^{\xi}=\overline{\mathbf{y}}^{\xi}-\hat{\mathbf{y}}^{\xi} \text {. }
$$

Case 2: $\underline{\text { Recalling of a pattern }} \mathbf{y}^{\xi}$ from an altered version of its key $\underline{\widetilde{\mathbf{x}}^{\xi}}$ :

1) Transform $\widetilde{\mathbf{x}}^{\xi}$ to $\mathbf{x}^{\xi}$ by applying the following transformation: $\mathbf{x}^{\xi}=\widetilde{\mathbf{x}}^{\xi}+\widehat{\mathbf{x}}^{\xi}$. 
2) Apply equations (6) and (7) to $\overline{\mathbf{x}}^{\xi}$ to get $\overline{\mathbf{y}}^{\xi}$, and

3) Anti-transform $\overline{\mathbf{y}}^{\xi}$ as $\mathbf{y}^{\xi}=\overline{\mathbf{y}}^{\xi}-\hat{\mathbf{y}}^{\xi}$ to get $\mathbf{y}^{\xi}$.

Example 3. Suppose we want to first memorize and then recall the following general fundamental set:

$$
\mathbf{x}^{1}=\left(\begin{array}{l}
0.1 \\
0.0 \\
0.2
\end{array}\right), \mathbf{y}^{1}=\left(\begin{array}{l}
1 \\
0 \\
0
\end{array}\right) ; \mathbf{x}^{2}=\left(\begin{array}{l}
0.4 \\
0.2 \\
0.5
\end{array}\right), \mathbf{y}^{2}=\left(\begin{array}{l}
0 \\
1 \\
0
\end{array}\right) \text { and } \mathbf{x}^{3}=\left(\begin{array}{l}
0.6 \\
0.6 \\
0.8
\end{array}\right), \mathbf{y}^{3}=\left(\begin{array}{l}
0 \\
0 \\
1
\end{array}\right)
$$

\section{TRAINING:}
1) $d=x_{1}^{2}-x_{1}^{1}=0.4-0.1=0.3$.
2) $\overline{\mathbf{x}}^{1}=\left(\begin{array}{lll}0.1 & 0.0 & 0.2\end{array}\right)^{T}$ and $\overline{\mathbf{y}}^{1}=\left(\begin{array}{lll}1 & 0 & 0\end{array}\right)^{T}$.
3) $\overline{\mathbf{x}}^{2}=\left(\begin{array}{l}0.4 \\ 0.3 \\ 0.5\end{array}\right), \hat{\mathbf{x}}^{2}=\left(\begin{array}{l}0.0 \\ 0.1 \\ 0.0\end{array}\right), \overline{\mathbf{y}}^{2}=\left(\begin{array}{l}1.3 \\ 0.3 \\ 0.3\end{array}\right), \hat{\mathbf{y}}^{2}=\left(\begin{array}{c}1.3 \\ -0.7 \\ 0.3\end{array}\right)$;

$$
\overline{\mathbf{x}}^{3}=\left(\begin{array}{l}
0.7 \\
0.6 \\
0.8
\end{array}\right), \hat{\mathbf{x}}^{3}=\left(\begin{array}{l}
0.1 \\
0.0 \\
0.0
\end{array}\right), \overline{\mathbf{y}}^{3}=\left(\begin{array}{c}
1.6 \\
0.6 \\
0.6
\end{array}\right), \hat{\mathbf{y}}^{3}=\left(\begin{array}{c}
1.6 \\
0.6 \\
-0.4
\end{array}\right) \text {. }
$$

You can easily show that: $\mathbf{M}=\mathbf{M}^{1}=\mathbf{M}^{2}=\mathbf{M}^{3}=\left(\begin{array}{lll}0.9 & 1.0 & 0.8 \\ -0.1 & 0.0 & -0.2 \\ -0.1 & 0.0 & -0.2\end{array}\right)$.

\section{RECALLING PHASE:}

Let us consider only Case 2, which is of more interest. Suppose we want to recall pattern $\mathbf{y}^{2}$ from its following distorted key: $\widetilde{\mathbf{x}}^{2}=\left(\begin{array}{lll}0.6 & 0.2 & 0.1\end{array}\right)^{T}$.

1) As discussed: $\mathbf{x}^{2}=\widetilde{\mathbf{x}}^{2}+\widehat{\mathbf{x}}^{2}=\left(\begin{array}{lll}0.6 & 0.3 & 0.1\end{array}\right)^{T}$.

$$
\begin{aligned}
& \text { 2) } \mathbf{M} \diamond_{\mathrm{B}} \mathbf{x}^{2}=\left(\begin{array}{ccc}
0.9 & 1.0 & 0.8 \\
-0.1 & 0.0 & -0.2 \\
-0.1 & 0.0 & -0.2
\end{array}\right) \diamond_{\mathrm{B}}\left(\begin{array}{l}
0.6 \\
0.3 \\
0.1
\end{array}\right)=\left(\begin{array}{c}
\operatorname{med}(1.5,1.3,0.9) \\
\operatorname{med}(0.5,0.3,-0.1) \\
\operatorname{med}(0.5,0.3,-0.1)
\end{array}\right)=\left(\begin{array}{l}
1.3 \\
0.3 \\
0.3
\end{array}\right) . \\
& \text { Finally, } \mathbf{y}^{2}=\overline{\mathbf{y}}^{2}-\hat{\mathbf{y}}^{2}=\left(\begin{array}{c}
1.3 \\
0.3 \\
0.3
\end{array}\right)-\left(\begin{array}{c}
1.3 \\
-0.7 \\
0.3
\end{array}\right)=\left(\begin{array}{l}
0 \\
1 \\
0
\end{array}\right) .
\end{aligned}
$$

\section{Experiments with Real Patterns}

The proposed associative memories were also tested with real patterns. We used the objects shown in Fig. 1. 

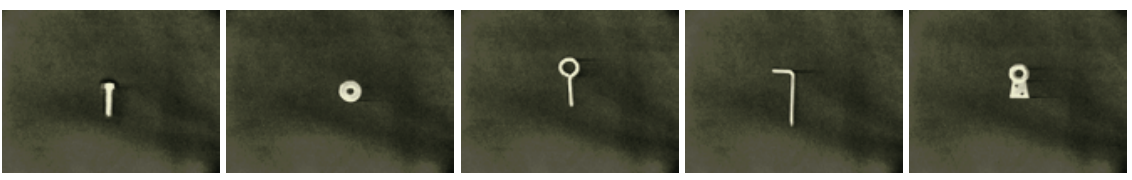

Fig. 1. The five objects used in the experiments. (a) A bolt. (b) A washer. (c) An eyebolt. (d) A hook. (e) A dovetail.

\subsection{Construction of the Association Matrix}

We did not directly recognize the objects by their images. We preferred to do it indirectly by invariant descriptions of each of them. For this, ninety images of each object in different positions, rotations and scale changes were captured. To each image a standard thresholder [5] was applied to get its binary version. Small spurious regions, due to bas thresholding, were eliminated form each image by means of a size filter [6, pp.47-48]. To each of the 19 images of each object (class) the first three Hu geometric invariants, to translations, rotations and scale changes were computed [7]. The five associations were built as:

$$
\begin{gathered}
\mathbf{x}^{1}=\left(\begin{array}{l}
0.4429 \\
0.1594 \\
0.0058
\end{array}\right), \mathbf{y}^{1}=\left(\begin{array}{l}
1 \\
0 \\
0 \\
0 \\
0
\end{array}\right) ; \mathbf{x}^{2}=\left(\begin{array}{l}
0.1896 \\
5.78 E-5 \\
4.14 E-6
\end{array}\right), \mathbf{y}^{2}=\left(\begin{array}{l}
0 \\
1 \\
0 \\
0 \\
0
\end{array}\right) ; \mathbf{x}^{3}=\left(\begin{array}{l}
0.7038 \\
0.2911 \\
0.1825
\end{array}\right), \mathbf{y}^{3}=\left(\begin{array}{l}
0 \\
0 \\
1 \\
0 \\
0
\end{array}\right) ; \\
\mathbf{x}^{4}=\left(\begin{array}{l}
1.1421 \\
1.5517 \\
0.8467
\end{array}\right), \mathbf{y}^{4}=\left(\begin{array}{l}
0 \\
0 \\
0 \\
1 \\
0
\end{array}\right) ; \mathbf{x}^{5}=\left(\begin{array}{l}
0.2491 \\
0.0195 \\
2.41 E-5
\end{array}\right), \mathbf{y}^{5}=\left(\begin{array}{l}
0 \\
0 \\
0 \\
0 \\
1
\end{array}\right) ;
\end{gathered}
$$

The three real numbers for each $\mathbf{x}^{\alpha}$ are the average values of the four Hu invariants computed with each set of 19 images of each object. The " 1 " at each $\mathbf{y}$ represents the index of the class of each object. After applying the methodology described in Section 4, matrix $\mathbf{M}$ is:

$$
\mathbf{M}=\left(\begin{array}{lll}
0.5571 & 0.8406 & 0.9942 \\
-0.4429 & -0.1594 & -0.0058 \\
-0.4429 & -0.1594 & -0.0058 \\
-0.4429 & -0.1594 & -0.0058 \\
-0.4429 & -0.1594 & -0.0058
\end{array}\right) .
$$

\subsection{Recalling of a Pattern by a Corrupted Version of Its Key}

In practical applications the noise added to the values of the patters rarely satisfies Theorem 2. To cope with this situation, we propose the following strategy: Once a general FS has been processed as described in Section 5, one of its patterns is classified in terms of a possible altered version of its key as follows: 


$$
\mathbf{y}^{j}, j=\underset{i}{\arg \min }\left(\min _{k=1}^{m}\left(\left|y_{k}-y_{k}^{i}\right|\right)\right)
$$

Table 1. Percentage of classification for the test set.

\begin{tabular}{|l|c|c|c|c|c|}
\cline { 2 - 6 } \multicolumn{1}{c|}{} & Bolt & Washer & Eyebolt & Hook & Dovetail \\
\hline Bolt & $100 \%$ & 0 & 0 & 0 & 0 \\
\hline Washer & 0 & $100 \%$ & 0 & 0 & 0 \\
\hline Eyebolt & $10 \%$ & 0 & $90 \%$ & 0 & 0 \\
\hline Hook & 0 & 0 & 0 & $100 \%$ & 0 \\
\hline Dovetail & 0 & $15 \%$ & 0 & 0 & $85 \%$ \\
\hline
\end{tabular}

Fifty images (10 for each object), and different from those used to build matrix $\mathbf{M}$ were used to measure the efficiency of the proposal. Of course the values of the invariants change. Table 1 shows the recalling results. As you can appreciate in $10 \%$ of the cases the eyebolt is classified as a bolt, and in $15 \%$ of the cases a dovetail is classified as a washer. In remaining cases the objects were correctly classified.

\section{Conclusions}

We have described a set of associative memories able to recall patterns altered by mixed noise. The proposed memories are based on the median operation. Numerical and real examples with images of real objects have been provided showing the performance of these memories. We gave the necessary and sufficient conditions under which the proposed memories are able to recall patterns either from the fundamental set of from altered versions of them. In this paper we show for the first time that associative memories combined with invariant features can be used to recognize objects in the presence of image transformations. Actually, we are investigating how to bypass the restricted conditions imposed by theorem 2 , and to avoid using equation (8).

\section{Acknowledgements}

This work was supported by CIC-IPN, COFAA-IPN, CONACYT under grants 41529 and 40114Y and CGPI-IPN under grant 20020658. We thank the reviewers for its comments for the improvement of this paper.

\section{References}

1. K. Steinbuch, Die Lernmatrix, Kybernetik, 1(1):26-45, 1961.

2. J. A. Anderson, A simple neural network generating an interactive memory, Mathematical Biosciences, 14:197-220, 1972.

3. J. J. Hopfield, Neural networks and physical systems with emergent collective computational abilities, Proceedings of the National Academy of Sciences, 79: 2554-2558, 1982.

4. G. X. Ritter el al. Morphological associative memories, IEEE Transactions on Neural Networks, 9:281-293, 1998.

5. N. Otsu, A threshold selection method from gray-level histograms, IEEE Transactions on SMC, 9(1): 62-66, 1979.

6. R. Jain et al. Machine Vision, McGraw-Hill, 1995.

7. M. K. Hu, Visual pattern recognition by moment invariants, IRE Transactions on Information Theory, 8: 179-187, 1962. 